\title{
The Structure of Parafermion Vertex Operator Algebras: General Case
}

\author{
Chongying Dong ${ }^{1,2, \star}$, Qing Wang ${ }^{3, \star \star}$ \\ 1 Department of Mathematics, University of California, Santa Cruz, CA 95064, USA \\ 2 School of Mathematics, Sichuan University, Chengdu 610065, China \\ 3 School of Mathematical Sciences, Xiamen University, Xiamen 361005, China. \\ E-mail: wangqing0793@gmail.com
}

Received: 15 September 2009 / Accepted: 26 March 2010

Published online: 15 August 2010 - (C) The Author(s) 2010. This article is published with open access at Springerlink.com

\begin{abstract}
The structure of the parafermion vertex operator algebra associated to an integrable highest weight module for any affine Kac-Moody algebra is studied. In particular, a set of generators for this algebra has been determined.
\end{abstract}

\section{Introduction}

This paper is a continuation of our study of the parafermion vertex operator algebra associated to an integrable highest weight module for an arbitrary affine Kac-Moody algebra. We determine a set of generators for these algebras. If the affine Kac-Moody algebra is $A_{1}^{(1)}$, this result was obtained previously in [6].

The parafermion algebra was first studied in [27] in the context of conformal field theory. It was clarified in [7] that the parafermion algebras are essentially the $Z$-algebras introduced and studied earlier in [19-21] in the process of studying the representation theory for the affine Kac-Moody Lie algebras. As it proved in [7], the parafermion algebras generate certain generalized vertex operator algebras. The partition functions for the parafermion conformal field theory have been given in [13] and [12] in connection with the partition functions associated to the integrable representations for the affine KacMoody Lie algebras. We refer the reader to [1,12,13,15,24,26,27] for various aspects of parafermion conformal field theory.

The parafermion vertex operator algebras which have roots in the parafermion conformal field theory are realized as commutants of the Heisenberg vertex operator subalgebras in the vertex operator algebras associated to the integrable highest weight modules for the affine Kac-Moody Lie algebra $[11,14,18]$. More precisely, let $L(k, 0)$ be the level $k$ integrable highest weight module for affine Kac-Moody algebra $\widehat{\mathfrak{g}}$ associated

\footnotetext{
* Supported by NSF grants, and a Faculty research grant from the University of California at Santa Cruz.

$\star \star$ Supported by China NSF grants (No.10931006, No.10926040), and Natural Science Foundation of Fujian Province, China (No.2009J05012).
} 
to a finite dimensional simple Lie algebra $\mathfrak{g}$. Then $L(k, 0)$ has a vertex operator subalgebra $M_{\widehat{\mathfrak{h}}}(k, 0)$ generated by the Cartan subalgebra $\mathfrak{h}$ of $\mathfrak{g}$. The commutant $K(\mathfrak{g}, k)$ of $M_{\widehat{\mathfrak{h}}}(k, 0)$ in $L(k, 0)$ is called the parafermion vertex operator algebra. Although the parafermion field theory has been studied for more than two decades, the mathematical investigation of the parafermion vertex operator algebras have been limited by a lack of understanding of the structural theory of these algebras. The goals of this paper and [4-6] are to alleviate this situation.

More importantly, it is widely believed that $K(\mathfrak{g}, k)$ should give a new class of rational, $C_{2}$-cofinite vertex operator algebras although this can only been proved in the case $\mathfrak{g}=s l_{2}$ and $k \leq 6$ [5]. Most well known vertex operator algebras such as lattice vertex operator algebras $[2,3,10]$, the affine vertex operator algebras $[7,11,23]$ and the Virasoro vertex operator algebras $[11,25]$ can be understood well using the underline lattices or Lie algebras. Unfortunately, the structures of parafermion vertex operator algebras with weight one subspaces being zero are much more complicated. It seems that a determination of a set of generators is the first step in understanding parafermion vertex operator algebras and their representation theory.

It is well known that $L(k, 0)$ is the irreducible quotient of the generalized Verma module $V(k, 0)$ (see Sect. 2). So the structure of $L(k, 0)$ can be determined by studying the maximal submodule of $V(k, 0)$ or the maximal ideal of $V(k, 0)$ which is also a vertex operator algebra. The same idea can also be applied to the study of parafermion vertex operator algebras. In fact, the Heisenberg vertex operator algebra $M_{\widehat{\mathfrak{h}}}(k, 0)$ is also a subalgebra of $V(k, 0)$ and the parafermion vertex operator algebra $K(\mathfrak{g}, k)$ is the simple quotient of the commutant $N(\mathfrak{g}, k)$ of $M_{\widehat{\mathfrak{h}}}(k, 0)$ in $V(k, 0)$. The main part of this paper is to determine a set of generators for $V(k, 0)(0)$ which is the weight zero subspace of $V(k, 0)$ under the action of the Cartan subalgebra $\mathfrak{h}$. The generators for $N(\mathfrak{g}, k)$ and $K(\mathfrak{g}, k)$ will be found easily then. Also, the maximal ideal of $N(\mathfrak{g}, k)$ is generated by one vector. This result is similar to that for the maximal ideal of $V(k, 0)$.

It is worth pointing out that the structure theory for the parafermion vertex operator algebra is similar to the structure theory for the finite dimensional Lie algebras or Kac-Moody Lie algebras. The building block of the Kac-Moody Lie algebras is the 3 -dimensional simple Lie algebra $s l_{2}$ associated to any real root. The generator results for the parafermion vertex operator algebras given in this paper and [6] show that the parafermion vertex operator algebras associated to the affine Lie algebra $A_{1}^{(1)}$ are also the building block of general parafermion vertex operator algebras. We hope this fact will be important in the future study of the representation theory for the parafermion vertex operator algebra. So a complete understanding of representation theory of $K(\mathfrak{g}, k)$ in the case $\mathfrak{g}=s l_{2}$ becomes necessary.

The paper is organized as follows. In Sect. 2, we give the construction of the vertex operator algebra $V(k, 0)$ associated to the affine Kac-Moody algebra $\widehat{\mathfrak{g}}$ from [11]. $V(k, 0)$ has a vertex operator subalgebra $V(k, 0)(0)$ which is the space of $\mathfrak{h}$-invariants of $V(k, 0)$. A foundational result in this section is to determine a set of of generators for $V(k, 0)(0)$. In Sect. 3, we give a set of generators for the vertex operator algebra $N(\mathfrak{g}, k)$ which is the commutant of the Heisenberg vertex operator algebra $M_{\widehat{\mathfrak{h}}}(k, 0)$ in $V(k, 0)$. We also discuss the vertex operator subalgebra $\widehat{P}_{\alpha}$ generated by $\omega_{\alpha}, W_{\alpha}^{3}$ (which is defined in Sect. 3) associated to any positive root $\alpha$ and prove that $\widehat{P}_{\alpha}$ is isomorphic to $N\left(s l_{2}, k_{\alpha}\right)$, where $k_{\alpha}=\frac{\langle\theta, \theta\rangle}{\langle\alpha, \alpha\rangle} k$ and $\theta$ is the highest root. In fact, $N(\mathfrak{g}, k)$ is generated by $\widehat{P}_{\alpha}$ for positive roots $\alpha$. In Sect. 4 , we give a set of generators for the parafermion vertex operator algebra $K(\mathfrak{g}, k)$ which is the simple quotient of $N(\mathfrak{g}, k)$. We prove that 
the maximal ideal of $N(\mathfrak{g}, k)$ is generated by the vector $x_{-\theta}(0)^{k+1} x_{\theta}(-1)^{k+1} \mathbb{1}$, where $x_{\theta}$ is the root vector associated to $\theta$ (see Sect. 2). We also show that the image $P_{\alpha}$ of $\widehat{P}_{\alpha}$ in $K(\mathfrak{g}, k)$ is isomorphic to $K\left(s l_{2}, k_{\alpha}\right)$ and $K(\mathfrak{g}, k)$ is generated by $P_{\alpha}$ for positive roots $\alpha$. That is, $K\left(s l_{2}, k_{\alpha}\right)$ are the building blocks of $K(\mathfrak{g}, k)$.

We expect the reader to be familiar with the elementary theory of vertex operator algebras as found, for example, in [10] and [18].

We thank the referees for many excellent suggestions which improve and simplify the proof of Theorem 2.1 greatly.

\section{Vertex Operator Algebras $V(k, 0)$ and $V(k, 0)(0)$}

Let $\mathfrak{g}$ be a finite dimensional simple Lie algebra with a Cartan subalgebra $\mathfrak{h}$. Let $\Delta$ be the corresponding root system and $Q$ the root lattice. Let $\langle$,$\rangle be an invariant symmetric$ nondegenerate bilinear form on $\mathfrak{g}$ such that $\langle\alpha, \alpha\rangle=2$ if $\alpha$ is a long root, where we have identified $\mathfrak{h}$ with $\mathfrak{h}^{*}$ via $\langle$,$\rangle . As in [15], we denote the image of \alpha \in \mathfrak{h}^{*}$ in $\mathfrak{h}$ by $t_{\alpha}$. That is, $\alpha(h)=\left\langle t_{\alpha}, h\right\rangle$ for any $h \in \mathfrak{h}$. Fix simple roots $\left\{\alpha_{1}, \cdots, \alpha_{l}\right\}$ and denote the highest root by $\theta$.

Let $\mathfrak{g}_{\alpha}$ denote the root space associated to the root $\alpha \in \Delta$. For $\alpha \in \Delta_{+}$, we fix $x_{ \pm \alpha} \in \mathfrak{g}_{ \pm \alpha}$ and $h_{\alpha}=\frac{2}{\langle\alpha, \alpha\rangle} t_{\alpha} \in \mathfrak{h}$ such that $\left[x_{\alpha}, x_{-\alpha}\right]=h_{\alpha},\left[h_{\alpha}, x_{ \pm \alpha}\right]= \pm 2 x_{ \pm \alpha}$. That is, $\mathfrak{g}^{\alpha}=\mathbb{C} x_{\alpha}+\mathbb{C} h_{\alpha}+\mathbb{C} x_{-\alpha}$ is isomorphic to $s l_{2}$ by sending $x_{\alpha}$ to $\left(\begin{array}{ll}0 & 1 \\ 0 & 0\end{array}\right), x_{-\alpha}$ to $\left(\begin{array}{ll}0 & 0 \\ 1 & 0\end{array}\right)$ and $h_{\alpha}$ to $\left(\begin{array}{ll}1 & 0 \\ 0 & -1\end{array}\right)$. Then $\left\langle h_{\alpha}, h_{\alpha}\right\rangle=2 \frac{\langle\theta, \theta\rangle}{\langle\alpha, \alpha\rangle}$ and $\left\langle x_{\alpha}, x_{-\alpha}\right\rangle=\frac{\langle\theta, \theta\rangle}{\langle\alpha, \alpha\rangle}$ for all $\alpha \in \Delta$.

Let $\widehat{\mathfrak{g}}=\mathfrak{g} \otimes \mathbb{C}\left[t, t^{-1}\right] \oplus \mathbb{C} K$ be the corresponding affine Lie algebra. Let $k \geq 1$ be an integer and

$$
V(k, 0)=V_{\widehat{\mathfrak{g}}}(k, 0)=\operatorname{Ind} \underset{\mathfrak{g} \otimes \mathbb{C}[t] \oplus \mathbb{C} K}{\widehat{\mathfrak{g}}} \mathbb{C}
$$

the induced $\widehat{\mathfrak{g}}$-module such that $\mathfrak{g} \otimes \mathbb{C}[t]$ acts as 0 and $K$ acts as $k$ on $\mathbb{1}=1$.

We denote by $a(n)$ the operator on $V(k, 0)$ corresponding to the action of $a \otimes t^{n}$. Then

$$
[a(m), b(n)]=[a, b](m+n)+m\langle a, b\rangle \delta_{m+n, 0} k
$$

for $a, b \in \mathfrak{g}$ and $m, n \in \mathbb{Z}$.

Let $a(z)=\sum_{n \in \mathbb{Z}} a(n) z^{-n-1}$. Then $V(k, 0)$ is a vertex operator algebra generated by $a(-1) \mathbb{1}$ for $a \in \mathfrak{g}$ such that $Y(a(-1) \mathbb{1}, z)=a(z)$ with the vacuum vector $\mathbb{1}$ and the Virasoro vector

$$
\omega_{\mathrm{aff}}=\frac{1}{2\left(k+h^{\vee}\right)}\left(\sum_{i=1}^{l} h_{i}(-1) h_{i}(-1) \mathbb{1}+\sum_{\alpha \in \Delta} \frac{\langle\alpha, \alpha\rangle}{\langle\theta, \theta\rangle} x_{\alpha}(-1) x_{-\alpha}(-1) \mathbb{1}\right)
$$

of central charge $\frac{k \operatorname{dim} \mathfrak{g}}{k+h^{\vee}}$ (e.g. [17,18, Sect. 6.2]), where $h^{\vee}$ is the dual Coxeter number of $\mathfrak{g}$ and $\left\{h_{i} \mid i=1, \ldots, l\right\}$ is an orthonormal basis of $\mathfrak{h}$.

We will use the standard notation for the component operators of $Y(u, z)$ for $u \in$ $V(k, 0)$. That is, $Y(u, z)=\sum_{n \in \mathbb{Z}} u_{n} z^{-n-1}$. From the definition of vertex operators, we immediately see that $(a(-1) \mathbb{1})_{n}=a(n)$ for $a \in \mathfrak{g}$. So in the rest of paper, we will 
use both $a(n)$ and $(a(-1) \mathbb{1})_{n}$ for $a \in \mathfrak{g}$ and use $u_{n}$ only for general $u$ without further explanation.

For $\lambda \in \mathfrak{h}^{*}$, set

$$
V(k, 0)(\lambda)=\{v \in V(k, 0) \mid h(0) v=\lambda(h) v, \forall h \in \mathfrak{h}\} .
$$

Then we have

$$
V(k, 0)=\oplus_{\lambda \in Q} V(k, 0)(\lambda) .
$$

Since $[h(0), Y(u, z)]=Y(h(0) u, z)$ for $h \in \mathfrak{h}$ and $u \in V(k, 0)$, from the definition of affine vertex operator algebra, we see that $V(k, 0)(0)$ is a vertex operator subalgebra of $V(k, 0)$ with the same Virasoro vector $\omega_{\text {aff }}$ and each $V(k, 0)(\lambda)$ is a module for $V(k, 0)(0)$.

Our first theorem is on a set of generators for $V(k, 0)(0)$.

Theorem 2.1. The vertex operator algebra $V(k, 0)(0)$ is generated by vectors $\alpha_{i}(-1) \mathbb{1}$ and $x_{-\alpha}(-2) x_{\alpha}(-1) \mathbb{1}$ for $1 \leq i \leq l, \alpha \in \Delta_{+}$.

Proof. First note that $V(k, 0)(0)$ is spanned by the vectors

$$
a_{1}\left(-m_{1}\right) \cdots a_{s}\left(-m_{s}\right) x_{\beta_{1}}\left(-n_{1}\right) x_{\beta_{2}}\left(-n_{2}\right) \cdots x_{\beta_{t}}\left(-n_{t}\right) \mathbb{1},
$$

where $a_{i} \in \mathfrak{h}, \beta_{j} \in \Delta, m_{i}>0, n_{j}>0$ and $\beta_{1}+\beta_{2}+\cdots+\beta_{t}=0$. Let $U$ be the vertex operator subalgebra generated by $\alpha_{i}(-1) \mathbb{1}$ and $x_{-\alpha}(-2) x_{\alpha}(-1) \mathbb{1}$ for $1 \leq i \leq l, \alpha \in$ $\Delta_{+}$. Clearly, $\alpha_{i}(-1) \mathbb{1}$ and $x_{-\alpha}(-2) x_{\alpha}(-1) \mathbb{1} \in V(k, 0)(0)$ for $1 \leq i \leq \bar{l}, \alpha \in \Delta_{+}$. It suffices to prove that $V(k, 0)(0) \subset U$.

Since $(h(-1) \mathbb{1})_{n}=h(n)$ for $h \in \mathfrak{h}$, we see that $h(n) U \subset U$ for $h \in \mathfrak{h}$ and $n \in \mathbb{Z}$. So we only need to prove $u=x_{\beta_{1}}\left(-n_{1}\right) x_{\beta_{2}}\left(-n_{2}\right) \cdots x_{\beta_{t}}\left(-n_{t}\right) \mathbb{1} \in U$ with $\beta_{1}+\beta_{2}+\cdots+\beta_{t}=0$. We will prove it by induction on $t$.

Clearly, $t \geq 2$. If $t=2$, it follows from Theorem 2.1 in [6] that

$$
x_{-\alpha}(-m) x_{\alpha}(-n) \mathbb{1} \in U
$$

for $m, n>0$. Note that if $m \geq 0$, then

$$
x_{-\alpha}(m) x_{\alpha}(n) \mathbb{1}=-h_{\alpha}(m+n) \mathbb{1}+m k\left\langle x_{\alpha}, x_{-\alpha}\right\rangle \delta_{m+n, 0} \mathbb{1} \in U .
$$

We claim that $x_{-\alpha}(m) x_{\alpha}(n) U \subset U$ for all $m, n \in \mathbb{Z}$. Let $u \in U$. From Proposition 4.5.7 of [18], there exist nonnegative integers $p, q$ such that

$$
x_{-\alpha}(m) x_{\alpha}(n) u=\sum_{i=0}^{p} \sum_{j=0}^{q}\left(\begin{array}{c}
m-q \\
i
\end{array}\right)\left(\begin{array}{l}
q \\
j
\end{array}\right)\left(x_{-\alpha}(m-q-i+j) x_{\alpha}(-1) \mathbb{1}\right)_{n+q+i-j} u .
$$

Since $x_{-\alpha}(m-q-i+j) x_{\alpha}(-1) \mathbb{1} \in U$, the claim follows.

From now on, we assume that $t>2$ and that

$$
x_{\beta_{1}}\left(-n_{1}\right) x_{\beta_{2}}\left(-n_{2}\right) \cdots x_{\beta_{v}}\left(-n_{v}\right) \mathbb{1} \in U
$$

with $\beta_{1}+\beta_{2}+\cdots+\beta_{v}=0$ for $2 \leq v \leq t-1$ and $n_{i}>0$. We have to show that

$$
x_{\beta_{1}}\left(-n_{1}\right) x_{\beta_{2}}\left(-n_{2}\right) \cdots x_{\beta_{t}}\left(-n_{t}\right) \mathbb{1} \in U
$$

with $\beta_{1}+\beta_{2}+\cdots+\beta_{t}=0$. We divide the proof into two cases. 
Case 1. There exist $1 \leq i, j \leq t$ such that $\beta_{i}+\beta_{j} \in \Delta$. Note that if

$$
x_{\beta_{1}}\left(-n_{1}\right) x_{\beta_{2}}\left(-n_{2}\right) \cdots x_{\beta_{t}}\left(-n_{t}\right) \mathbb{1} \in U
$$

then

$$
x_{\beta_{i_{1}}}\left(-n_{i_{1}}\right) x_{\beta_{i_{2}}}\left(-n_{i_{2}}\right) \cdots x_{\beta_{i_{t}}}\left(-n_{i_{t}}\right) \mathbb{1} \in U
$$

by the induction assumption, where $\left(i_{1}, \ldots, i_{t}\right)$ is any permutation of $(1, \ldots, t)$. Without loss of generality, we may assume that $\beta_{1}+\beta_{2} \in \Delta$.

Let $k, n$ be positive integers such that $-k+n=-n_{2}$ and $n>n_{i}$ for $i \geq 3$. Let $w=x_{\beta_{1}+\beta_{2}}(-k) x_{\beta_{3}}\left(-n_{3}\right) \cdots x_{\beta_{t}}\left(-n_{t}\right) \mathbb{1}$ with $\beta_{1}+\beta_{2}+\cdots+\beta_{t}=0$. Then $w \in U$ by the induction assumption and $x_{\beta_{1}}\left(-n_{1}\right) x_{-\beta_{1}}(n) w \in U$ by the claim.

Let $\left[x_{-\beta_{1}}, x_{\beta_{1}+\beta_{2}}\right]=\lambda x_{\beta_{2}}$ for some nonzero $\lambda$. Then

$$
\begin{aligned}
& x_{\beta_{1}}\left(-n_{1}\right) x_{-\beta_{1}}(n) w=\lambda x_{\beta_{1}}\left(-n_{1}\right) x_{\beta_{2}}\left(-n_{2}\right) x_{\beta_{3}}\left(-n_{3}\right) \cdots x_{\beta_{t}}\left(-n_{t}\right) \mathbb{1} \\
& \quad+x_{\beta_{1}}\left(-n_{1}\right) x_{\beta_{1}+\beta_{2}}(-k)\left[x_{-\beta_{1}}, x_{\beta_{3}}\right]\left(n-n_{3}\right) x_{\beta_{4}}\left(-n_{4}\right) \cdots x_{\beta_{t}}\left(-n_{t}\right) \mathbb{1} \\
& \quad+\cdots+x_{\beta_{1}}\left(-n_{1}\right) x_{\beta_{1}+\beta_{2}}(-k) x_{\beta_{3}}\left(-n_{3}\right) \cdots\left[x_{-\beta_{1}}, x_{\beta_{t}}\right]\left(n-n_{t}\right) \mathbb{1} .
\end{aligned}
$$

Since $n-n_{i}>0$ for $i \geq 3$, we see that

$$
\begin{aligned}
& x_{\beta_{1}}\left(-n_{1}\right) x_{\beta_{1}+\beta_{2}}(-k)\left[x_{-\beta_{1}}, x_{\beta_{3}}\right]\left(n-n_{3}\right) x_{\beta_{4}}\left(-n_{4}\right) \cdots x_{\beta_{t}}\left(-n_{t}\right) \mathbb{1} \\
& +\cdots+x_{\beta_{1}}\left(-n_{1}\right) x_{\beta_{1}+\beta_{2}}(-k) x_{\beta_{3}}\left(-n_{3}\right) \cdots\left[x_{-\beta_{1}}, x_{\beta_{t}}\right]\left(n-n_{t}\right) \mathbb{1}
\end{aligned}
$$

lies in $U$ by the induction assumption. As a result, $x_{\beta_{1}}\left(-n_{1}\right) x_{\beta_{2}}\left(-n_{2}\right) x_{\beta_{3}}\left(-n_{3}\right)$ $\cdots x_{\beta_{t}}\left(-n_{t}\right) \mathbb{1} \in U$.

Case 2. For any $1 \leq i, j \leq t, \beta_{i}+\beta_{j} \notin \Delta$. We claim that there exist $1 \leq i^{\prime}, j^{\prime} \leq t$ such that $\beta_{i^{\prime}}+\beta_{j^{\prime}}=0$. Otherwise, $\beta_{i}+\beta_{j} \neq 0$ for all $i, j$. This implies that $\left\langle\beta_{i}, \beta_{j}\right\rangle \geq 0$ for all $i, j$, thus $\left\langle\beta_{1}, \sum_{j=2}^{t} \beta_{j}\right\rangle \geq 0$. On the other hand, since $\sum_{j=2}^{t} \beta_{j}=-\beta_{1}$, we have $\left\langle\beta_{1}, \sum_{j=2}^{t} \beta_{j}\right\rangle<0$, a contradiction.

Without loss of generality, we may assume $\beta_{1}+\beta_{2}=0$. Then $\beta_{3}+\cdots+\beta_{t}=0$. By the induction assumption, $x_{\beta_{3}}\left(-n_{3}\right) \cdots x_{\beta_{t}}\left(-n_{t}\right) \mathbb{1} \in U$. It is immediate that

$$
x_{\beta_{1}}\left(-n_{1}\right) x_{\beta_{2}}\left(-n_{2}\right) x_{\beta_{3}}\left(-n_{3}\right) \cdots x_{\beta_{t}}\left(-n_{t}\right) \mathbb{1} \in U
$$

from the claim. The proof is complete.

Remark 2.2. Theorem 2.1 has been obtained in [6] previously in the case $\mathfrak{g}=s l_{2}$. The proof given here simplifies the proof of Theorem 2.1 in [6].

Next we discuss some automorphisms of vertex operator algebras $V(k, 0)$ and $V(k, 0)(0)$ for later purposes. It is well known that the automorphism group $\operatorname{Aut}(V(k, 0))$ is isomorphic to the automorphism group $\operatorname{Aut}(\mathfrak{g})$. In fact, if $\sigma \in \operatorname{Aut}(\mathfrak{g})$, then $\sigma$ lifts to an automorphism of $V(k, 0)$ in the following way:

$$
\sigma\left(x_{1}\left(-n_{1}\right) \cdots x_{s}\left(-n_{s}\right) \mathbb{1}\right)=\left(\sigma x_{1}\right)\left(-n_{1}\right) \cdots\left(\sigma x_{s}\right)\left(-n_{s}\right) \mathbb{1}
$$

for $x_{i} \in \mathfrak{g}$ and $n_{i}>0$. Let $W(\mathfrak{g})$ be the Weyl group of $\mathfrak{g}$. Then $W(\mathfrak{g})$ can naturally be regarded as a subgroup of $\operatorname{Aut}(\mathfrak{g})$ [16]. It is easy to see that if $\sigma(\mathfrak{h})=\mathfrak{h}$, then $\sigma(V(k, 0)(0))=V(k, 0)(0)$ and the restriction of $\sigma$ to $V(k, 0)(0)$ gives an automorphism of $V(k, 0)(0)$. In particular, any Weyl group element gives an automorphism of $V(k, 0)(0)$. This fact will be used in later sections. 


\section{Vertex Operator Algebra $N(\mathfrak{g}, k)$}

Let $V_{\widehat{\mathfrak{h}}}(k, 0)$ be the vertex operator subalgebra of $V(k, 0)$ generated by $h(-1) \mathbb{1}$ for $h \in \mathfrak{h}$ with the Virasoro element

$$
\omega_{\mathfrak{h}}=\frac{1}{2 k} \sum_{i=1}^{l} h_{i}(-1) h_{i}(-1) \mathbb{1}
$$

of central charge $l$, where $\left\{h_{1}, \cdots h_{l}\right\}$ is an orthonormal basis of $\mathfrak{h}$ as before. For $\lambda \in \mathfrak{h}^{*}$, let $M_{\widehat{\mathfrak{h}}}(k, \lambda)$ denote the irreducible highest weight module for $\widehat{\mathfrak{h}}$ with a highest weight vector $v_{\lambda}$ such that $h(0) v_{\lambda}=\lambda(h) v_{\lambda}$ for $h \in \mathfrak{h}$. Then $V_{\widehat{\mathfrak{h}}}(k, 0)$ is identified with $M_{\widehat{\mathfrak{h}}}(k, 0)$.

Recall $V(k, 0)(\lambda)$ from Sect. 2. Both $V(k, 0)$ and $V(k, 0)(\lambda), \lambda \in Q$ are completely reducible $V_{\widehat{\mathfrak{h}}}(k, 0)$-modules. That is,

$$
\begin{aligned}
V(k, 0) & =\oplus_{\lambda \in Q} M_{\widehat{\mathfrak{h}}}(k, \lambda) \otimes N_{\lambda}, \\
V(k, 0)(\lambda) & =M_{\widehat{\mathfrak{h}}}(k, \lambda) \otimes N_{\lambda},
\end{aligned}
$$

where

$$
N_{\lambda}=\left\{v \in V(k, 0) \mid h(m) v=\lambda(h) \delta_{m, 0} v \text { for } h \in \mathfrak{h}, m \geq 0\right\}
$$

is the space of highest weight vectors with highest weight $\lambda$ for $\widehat{\mathfrak{h}}$.

Note that $N(\mathfrak{g}, k)=N_{0}$ is the commutant [11, Theorem 5.1] of $V_{\widehat{\mathfrak{h}}}(k, 0)$ in $V(k, 0)$. The commutant $N(\mathfrak{g}, k)$ is a vertex operator algebra with the Virasoro vector $\omega=$ $\omega_{\text {aff }}-\omega_{\mathfrak{h}}$ whose central charge is $\frac{k \operatorname{dim} \mathfrak{g}}{k+h^{\vee}}-l$.

Recall from Sect. 2, the 3-dimensional subalgebra $\mathfrak{g}^{\alpha}$ for $\alpha \in \Delta_{+}$. Then the restriction $\langle,\rangle_{\mathfrak{g}^{\alpha}}$ of the bilinear form $\langle$,$\rangle to \mathfrak{g}^{\alpha}$ is equal to $\frac{\langle\theta, \theta\rangle}{\langle\alpha, \alpha\rangle}($,$) , where ($,$) is the standard$ nondegenerate symmetric invariant bilinear form on $\mathfrak{g}^{\alpha}$ such that $\left(h_{\alpha}, h_{\alpha}\right)=2$. As a result, $V(k, 0)$ is a module for $\widehat{\mathfrak{g}}^{\alpha}=\mathfrak{g}^{\alpha} \otimes \mathbb{C}\left[t, t^{-1}\right] \oplus \mathbb{C} K$ of level $k_{\alpha}=\frac{\langle\theta, \theta\rangle}{\langle\alpha, \alpha\rangle} k$ as we regard $V(k, 0)$ as a module for the subalgebra $\widehat{\mathfrak{g}}^{\alpha}$ of $\widehat{\mathfrak{g}}$. In other words, $V(k, 0)$ is a $\mathfrak{g}^{\alpha}$-module of level $2 k$ or $3 k$ if $\alpha$ is a short root.

Following [5], we let

$$
\begin{gathered}
\omega_{\alpha}=\frac{1}{2 k(k+2)}\left(-k h_{\alpha}(-2) \mathbb{1}-h_{\alpha}(-1)^{2} \mathbb{1}+2 k x_{\alpha}(-1) x_{-\alpha}(-1) \mathbb{1}\right), \\
W_{\alpha}^{3}=k^{2} h_{\alpha}(-3) \mathbb{1}+3 k h_{\alpha}(-2) h_{\alpha}(-1) \mathbb{1}+2 h_{\alpha}(-1)^{3} \mathbb{1} \\
-6 k h_{\alpha}(-1) x_{\alpha}(-1) x_{-\alpha}(-1) \mathbb{1} \\
+3 k^{2} x_{\alpha}(-2) x_{-\alpha}(-1) \mathbb{1}-3 k^{2} x_{\alpha}(-1) x_{-\alpha}(-2) \mathbb{1}
\end{gathered}
$$

if $\alpha \in \triangle_{+}$is a long root. If $\alpha$ is a short root, we also define $\omega_{\alpha}, W_{\alpha}^{3}$ as in (3.3) and (3.4) by replacing $k$ by $k_{\alpha}$.

Let $\widehat{P}_{\alpha}$ be the vertex operator subalgebra of $N(\mathfrak{g}, k)$ generated by $\omega_{\alpha}$ and $W_{\alpha}^{3}$. Then $\widehat{P}_{\alpha}$ is isomorphic to the $W$-algebra $W(2,3,4,5)$ [1] by [6, Theorem 3.1] with $k$ replaced by $k_{\alpha}$, i.e., $\widehat{P}_{\alpha}$ is isomorphic to $N\left(s l_{2}, k_{\alpha}\right)$.

The first main theorem of this paper is about the generators of $N(\mathfrak{g}, k)$.

Theorem 3.1. The vertex operator algebra $N(\mathfrak{g}, k)$ is generated by $\operatorname{dim} \mathfrak{g}-l$ vectors $\omega_{\alpha}$ and $W_{\alpha}^{3}$ for $\alpha \in \Delta_{+}$. That is, $N(\mathfrak{g}, k)$ is generated by subalgebras $\widehat{P}_{\alpha}$ for $\alpha \in \Delta_{+}$. 
Proof. We first prove that $V(k, 0)(0)$ is generated by vectors $\alpha_{i}(-1) \mathbb{1}, \omega_{\alpha}$ and $W_{\alpha}^{3}$ for $i=1, \ldots, l$ and $\alpha \in \Delta_{+}$. In fact, let $U$ be the vertex operator subalgebra generated by $h(-1) \mathbb{1}, \omega_{\alpha}$ and $W_{\alpha}^{3}$ for $h \in \mathfrak{h}$ and $\alpha \in \Delta_{+}$. Then $x_{-\alpha}(-1) x_{\alpha}(-1) \mathbb{1} \in U$ and $\omega_{\text {aff }} \in U$. Moreover, from the expression of $W_{\alpha}^{3}$, we see that $x_{-\alpha}(-1) x_{\alpha}(-2) \mathbb{1}-$ $x_{-\alpha}(-2) x_{\alpha}(-1) \mathbb{1} \in U$. Set $L_{\text {aff }}(n)=\left(\omega_{\text {aff }}\right)_{n+1}$; we have

$$
\left[L_{\mathrm{aff}}(m), a(n)\right]=-n a(m+n)
$$

for $m, n \in \mathbb{Z}, a \in \mathfrak{g}$. Thus,

$$
L_{\text {aff }}(-1) x_{-\alpha}(-1) x_{\alpha}(-1) \mathbb{1}=x_{-\alpha}(-2) x_{\alpha}(-1) \mathbb{1}+x_{-\alpha}(-1) x_{\alpha}(-2) \mathbb{1} \in U .
$$

Together with $x_{-\alpha}(-1) x_{\alpha}(-2) \mathbb{1}-x_{-\alpha}(-2) x_{\alpha}(-1) \mathbb{1} \in U$, we get $x_{-\alpha}(-2) x_{\alpha}(-1) \mathbb{1} \in$ $U$, and so $U$ is equal to $V(k, 0)(0)$ by Theorem 2.1 .

Next we show that $\omega_{\alpha}, W_{\alpha}^{3} \in N(\mathfrak{g}, k)$ for $\alpha \in \Delta$. Since $\left\langle h_{\alpha}, h_{\alpha}\right\rangle \neq 0$, we have decomposition $\mathfrak{h}=\mathbb{C} h_{\alpha} \oplus\left(\mathbb{C} h_{\alpha}\right)^{\perp}$, where $\left(\mathbb{C} h_{\alpha}\right)^{\perp}$ is the orthogonal complement of $\mathbb{C} h_{\alpha}$ with respect to $\langle$,$\rangle . From [6], we know that h_{\alpha}(n) \omega_{\alpha}=h_{\alpha}(n) W_{\alpha}^{3}=0$ for $n \geq 0$. If $u \in\left(\mathbb{C} h_{\alpha}\right)^{\perp}$, we clearly have $u(n) \omega_{\alpha}=u(n) W_{\alpha}^{3}=0$ for $n \geq 0$. This implies that $\omega_{\alpha}, W_{\alpha}^{3} \in N(\mathfrak{g}, k)$.

Notice that $Y\left(u, z_{1}\right) Y\left(v, z_{2}\right)=Y\left(v, z_{2}\right) Y\left(u, z_{1}\right)$ for $u \in M_{\widehat{h}}(k, 0)$ and $v \in N(\mathfrak{g}, k)$. Since $V(k, 0)(0)=M_{\widehat{\mathfrak{h}}}(k, 0) \otimes N(\mathfrak{g}, k), h(-1) \mathbb{1} \in M_{\widehat{\mathfrak{h}}}(k, 0)$ for $h \in \mathfrak{h}$, and $\omega_{\alpha}, W_{\alpha}^{3} \in$ $N(\mathfrak{g}, k)$ for $\alpha \in \Delta_{+}$, we conclude that $N(\mathfrak{g}, k)$ is generated by $\omega_{\alpha}, W_{\alpha}^{3}$ for $\alpha \in \Delta_{+}$.

Remark 3.2. Using the $Z$-algebra introduced and studied in [19] and [20,21], we can rewrite $\omega_{\alpha}$ and $W_{\alpha}^{3}$ in terms of $Z$-operators $Z_{\alpha}(m)$ and $Z_{-\alpha}(n)$. It is not too hard to see that $\omega_{\alpha}=a_{\alpha} Z_{\alpha}(-1) Z_{-\alpha}(-1) \mathbb{1}$ and $W_{\alpha}^{3}=b_{\alpha} Z_{\alpha}(-2) Z_{-\alpha}(-1) \mathbb{1}+c_{\alpha} Z_{-\alpha}(-2) Z_{\alpha}(-1) \mathbb{1}$ for some constants $a_{\alpha}, b_{\alpha}, c_{\alpha} \in \mathbb{C}$. One could determine these constants explicitly using the definition of $Z$-operators.

Remark 3.3. The vertex operator algebra $N(\mathfrak{g}, k)$ and its quotient $K(\mathfrak{g}, k)$ are of moonshine type. That is, their weight zero subspaces are 1-dimensional and weight one subspaces are zero.

Remark 3.4. We already know that each $\omega_{\alpha}$ is a Virasoro element and how to compute the Lie brackets $\left[Y\left(\omega_{\alpha}, z_{1}\right), Y\left(W_{\alpha}^{3}, z_{2}\right)\right],\left[Y\left(W_{\alpha}^{3}, z_{1}\right), Y\left(W_{\alpha}^{3}, z_{2}\right)\right]$ for $\alpha \in \Delta_{+}$. It is important to calculate the Lie brackets for vertex operators associated to vectors in different $\widehat{P_{\alpha}}$. This will be done in a sequel to this paper where the representation theory will be investigated.

Following the discussion given at the end of Sect. 2, we see that any Weyl group element gives an automorphism of $N(\mathfrak{g}, k)$.

\section{Parafermion Vertex Operator Algebras $K(\mathfrak{g}, k)$}

It is well known that the vertex operator algebra $V(k, 0)$ has a unique maximal ideal $\mathcal{J}$ generated by a weight $k+1$ vector $x_{\theta}(-1)^{k+1} \mathbb{1}[17]$, where $\theta$ is the highest root of $\mathfrak{g}$. The quotient vertex operator algebra $L(k, 0)=V(k, 0) / \mathcal{J}$ is a simple, rational vertex operator algebra associated to the affine Lie algebra $\widehat{\mathfrak{g}}$. Again, the Heisenberg vertex operator algebra $V_{\widehat{h}}(k, 0)$ generated by $h(-1) \mathbb{1}$ for $h \in \mathfrak{h}$ is a simple subalgebra of $L(k, 0)$ and $L(k, 0)$ is a completely reducible $V_{\widehat{\mathfrak{h}}}(k, 0)$-module. We have a decomposition

$$
L(k, 0)=\bigoplus_{\lambda \in Q} M_{\widehat{\mathfrak{h}}}(k, \lambda) \otimes K_{\lambda}
$$


as modules for $V_{\widehat{\mathfrak{h}}}(k, 0)$, where

$$
K_{\lambda}=\left\{v \in L(k, 0) \mid h(m) v=\lambda(h) \delta_{m, 0} v \text { for } h \in \mathfrak{h}, m \geq 0\right\} .
$$

Set $K(\mathfrak{g}, k)=K_{0}$. Then $K(\mathfrak{g}, k)$ is the commutant of $V_{\widehat{\mathfrak{h}}}(k, 0)$ in $L(k, 0)$ and is called the parafermion vertex operator algebra associated to the irreducible highest weight module $L(k, 0)$ for $\widehat{\mathfrak{g}}$. As we mentioned in the Introduction, $K(\mathfrak{g}, k)$ are conjectured to be rational, $C_{2}$-cofinite vertex operator algebras.

As a $V_{\widehat{\mathfrak{h}}}(k, 0)$-module, $\mathcal{J}$ is completely reducible. From (3.1),

$$
\mathcal{J}=\oplus_{\lambda \in Q} M_{\widehat{\mathfrak{h}}}(k, \lambda) \otimes\left(\mathcal{J} \cap N_{\lambda}\right) .
$$

In particular, $\widetilde{\mathcal{I}}=\mathcal{J} \cap N(\mathfrak{g}, k)$ is an ideal of $N(\mathfrak{g}, k)$ and $K(\mathfrak{g}, k) \cong N(\mathfrak{g}, k) / \widetilde{\mathcal{I}}$. Following the same proof as [5, Lemma 3.1], we know that $\widetilde{\mathcal{I}}$ is the unique maximal ideal of $N(\mathfrak{g}, k)$. Thus $K(\mathfrak{g}, k)$ is a simple vertex operator algebra.

We still use $\omega_{\text {aff }}, \omega_{\mathfrak{h}}, \omega_{\alpha}, W_{\alpha}^{3}$ to denote their images in $L(k, 0)=V(k, 0) / \mathcal{J}$.

Remark 4.1. In the case $k=1$, it follows from the construction of $L(1,0)[9,10]$ that $\omega=0$ and $K(\mathfrak{g}, k)=\mathbb{C}$ if $\mathfrak{g}$ is of ADE type.

The following result is a direct consequence of Theorem 3.1.

Theorem 4.2. The simple vertex operator algebra $K(\mathfrak{g}, k)$ is generated by $\omega_{\alpha}, W_{\alpha}^{3}$ for $\alpha \in \Delta_{+}$.

Next, we study the ideal $\widetilde{\mathcal{I}}$ of $N(\mathfrak{g}, k)$ in detail. The vector $x_{\theta}(-1)^{k+1} \mathbb{1} \notin N(\mathfrak{g}, k)$. From [5, Theorem 3.2] we know that $h_{\theta}(n) x_{-\theta}(0)^{k+1} x_{\theta}(-1)^{k+1} \mathbb{1}=0$ for $n \geq 0$. It is clear that if $h \in \mathfrak{h}$ satisfies $\left\langle h_{\theta}, h\right\rangle=0$, then $h(n) x_{-\theta}(0)^{k+1} x_{\theta}(-1)^{k+1} \mathbb{1}=0$ for $n \geq 0$. So we have proved the following

Lemma 4.3. $x_{-\theta}(0)^{k+1} x_{\theta}(-1)^{k+1} \mathbb{1} \in \widetilde{\mathcal{I}}$.

Furthermore, we have

Proposition 4.4. The maximal ideal $\tilde{\mathcal{I}}$ of $N(\mathfrak{g}, k)$ is generated by $x_{-\theta}(0)^{k+1} x_{\theta}(-1)^{k+1} \mathbb{1}$.

Proof. The proof is similar to that of [6, Theorem 4.2 (1)]. Recall $\mathfrak{g}^{\theta}=\mathbb{C} x_{\theta}+\mathbb{C} h_{\theta}+$ $\mathbb{C} x_{-\theta}$ is a subalgebra of $\mathfrak{g}$ isomorphic to $s l_{2} . V(k, 0)$ is an $\mathfrak{g}^{\theta}$-module where $a \in \mathfrak{g}^{\theta}$ acts as $a(0)$. Each weight subspace of the vertex operator algebra $V(k, 0)$ is a finite dimensional $\mathfrak{g}^{\theta}$-module and $V(k, 0)$ is completely reducible as a module for $\mathfrak{g}^{\theta}$. Consider the $\mathfrak{g}^{\theta}$-submodule $X$ of $V(k, 0)$ generated by $x_{\theta}(-1)^{k+1} \mathbb{1}$. Since $x_{\theta}(0) x_{\theta}(-1)^{k+1} \mathbb{1}=0$ and $h_{\theta}(0) x_{\theta}(-1)^{k+1} \mathbb{1}=2(k+1) x_{\theta}(-1)^{k+1} \mathbb{1}, x_{\theta}(-1)^{k+1} \mathbb{1}$ is a highest weight vector with highest weight $2(k+1)$ for $\mathfrak{g}^{\theta}$. Then $X$ is an irreducible $\mathfrak{g}^{\theta}$-module with basis $x_{-\theta}(0)^{i} x_{\theta}(-1)^{k+1} \mathbb{1}, 0 \leq i \leq 2(k+1)$ from the representation theory of $s l_{2}$. This implies that the ideal $\mathcal{J}$ of the vertex operator algebra $V(k, 0)$ can be generated by any nonzero vector in $X$. In particular, $\mathcal{J}$ is generated by $x_{-\theta}(0)^{k+1} x_{\theta}(-1)^{k+1} \mathbb{1}$. Then $\mathcal{J}$ is spanned by $u_{n} x_{-\theta}(0)^{k+1} x_{\theta}(-1)^{k+1} \mathbb{1}$ for $u \in V(k, 0)$ and $n \in \mathbb{Z}$ by [8, Cor. 4.2] or [22, Prop. 4.1].

Since $v_{m} u \in V(k, 0)(\lambda+\mu)$ for $v \in V(k, 0)(\lambda), u \in V(k, 0)(\mu), \lambda, \mu \in Q$ and $m \in$ $\mathbb{Z}$, we see that $\mathcal{J} \cap V(k, 0)(0)$ is spanned by vectors of the form $u_{n} x_{-\theta}(0)^{k+1} x_{\theta}(-1)^{k+1} \mathbb{1}$ with $u \in V(k, 0)(0)$. Let $u=v \otimes w \in V(k, 0)(0)=M_{\widehat{\mathfrak{h}}}(k, 0) \otimes N(\mathfrak{g}, k)$ with $v \in M_{\widehat{\mathfrak{h}}}(k, 0)$ and $w \in N(\mathfrak{g}, k)$. Then $Y(u, z)=Y(v, z) \otimes Y(w, z)$ acts on $M_{\widehat{\mathfrak{h}}}(k, 0) \otimes$ $N(\mathfrak{g}, k)$. As a result, we have that $\widetilde{\mathcal{I}}$ is spanned by $w_{n} x_{-\theta}(0)^{k+1} x_{\theta}(-1)^{k+1} \mathbb{1}$ for $w \in$ $N(\mathfrak{g}, k)$ and $n \in \mathbb{Z}$. That is, the ideal $\widetilde{\mathcal{I}}$ of the vertex operator algebra $N(\mathfrak{g}, k)$ is generated by $x_{-\theta}(0)^{k+1} x_{\theta}(-1)^{k+1} \mathbb{1}$. The proof is complete. 
For $\alpha \in \Delta_{+}$, we let $P_{\alpha}$ be the vertex operator subalgebra of $K(\mathfrak{g}, k)$ generated by $\omega_{\alpha}$ and $W_{\alpha}^{3}$. Then $P_{\alpha}$ is a quotient of $\widehat{P}_{\alpha}$. A natural question is whether or not $P_{\alpha}$ is a simple vertex operator algebra. For this purpose, we recall our discussion earlier on the automorphisms of the vertex operator algebra $V(k, 0)$ and $N(\mathfrak{g}, k)$. That is, any Weyl group element gives an automorphism of $V(k, 0)$ and $N(\mathfrak{g}, k)$.

Clearly, any automorphism $\sigma$ of $V(k, 0)$ induces an automorphism of $L(k, 0)$ as $\sigma$ maps the unique maximal ideal $\mathcal{J}$ to $\mathcal{J}$. If $\sigma \in W(\mathfrak{g})$, then $\sigma$ preserves the unique maximal ideal $\widetilde{\mathcal{I}}$ and $\sigma$ gives an automorphism of the parafermion vertex operator algebra $K(\mathfrak{g}, k)$, Now let $\alpha \in \Delta_{+}$be a long root. Then there exists $\sigma \in W(\mathfrak{g})$ such that $\sigma \theta=\alpha$ [16]. As a result,

$$
\sigma\left(x_{-\theta}(0)^{k+1} x_{\theta}(-1)^{k+1} \mathbb{1}\right)=a x_{-\alpha}(0)^{k+1} x_{\alpha}(-1)^{k+1} \mathbb{1}
$$

for some constant $a$. This implies from Lemma 4.3 that $x_{-\alpha}(0)^{k+1} x_{\alpha}(-1)^{k+1} \mathbb{1} \in \widetilde{\mathcal{I}}$. Using [6, Theorem 4.2] we obtain:

Proposition 4.5. For any long root $\alpha \in \Delta_{+}$, the vertex operator subalgebra $P_{\alpha}$ of $K(\mathfrak{g}, k)$ is a simple vertex operator algebra isomorphic to the parafermion vertex operator algebra $\mathrm{K}\left(\mathrm{sl}_{2}, \mathrm{k}\right)$ associated to $\mathrm{sl}_{2}$.

We next deal with short roots $\alpha \in \Delta_{+}$. As we mentioned already $V(k, 0)$ is a level $k_{\alpha}$-module for the affine algebra $\widehat{\mathfrak{g}}^{\alpha}$. We need a different method to prove the following which is a generalization of Proposition 4.5.

Proposition 4.6. Let $\alpha \in \Delta_{+}$. Then the vertex operator subalgebra $P_{\alpha}$ of $K(\mathfrak{g}, k)$ is a simple vertex operator algebra isomorphic to the parafermion vertex operator algebra $K\left(s l_{2}, k_{\alpha}\right)$ associated to $\mathrm{sl}_{2}$.

Proof. As in the proof of Proposition 4.5 we only need to prove that

$$
x_{-\alpha}(0)^{k_{\alpha}+1} x_{\alpha}(-1)^{k_{\alpha}+1} \mathbb{1} \in \widetilde{\mathcal{I}} .
$$

Clearly, $L(k, 0)$ is an integrable module for $\widehat{\mathfrak{g}}^{\alpha}$ as $x_{\alpha}(-1)$ is locally nilpotent on $L(k, 0)$. In particular, the vertex operator subalgebra $U$ of $L(k, 0)$ generated by $\mathfrak{g}^{\alpha}$ is an integrable highest weight module. That is, $U$ is isomorphic to $L\left(k_{\alpha}, 0\right)$ associated to the affine algebra $\widehat{\mathfrak{g}}^{\alpha}$. As a result, we have $x_{\alpha}(-1)^{k_{\alpha}+1} \mathbb{1} \in \mathcal{J}$. It follows then immediately that $x_{-\alpha}(0)^{k_{\alpha}+1} x_{\alpha}(-1)^{k_{\alpha}+1} \mathbb{1} \in \widetilde{\mathcal{I}}$, as desired.

Remark 4.7. We expect from Proposition 4.6 that the role of $K\left(s l_{2}, k_{\alpha}\right)$ played in the theory of the parafermion vertex operator algebra is similar to the role of $s l_{2}$ played in the theory of Kac-Moody Lie algebras. So a study of structural and representation theory for $K\left(s l_{2}, k_{\alpha}\right)$ becomes extremely important for general parafermion vertex operator algebras.

Open Access This article is distributed under the terms of the Creative Commons Attribution Noncommercial License which permits any noncommercial use, distribution, and reproduction in any medium, provided the original author(s) and source are credited.

\section{References}

1. Blumenhagen, R., Eholzer, W., Honecker, A., Hornfeck, K., Hübel, R.: Coset realization of unifying $W$-algebras. Int. J. Mod. Phys. A10, 2367-2430 (1995) 
2. Borcherds, R.E.: Vertex algebras, Kac-Moody algebras, and the Monster. Proc. Natl. Acad. Sci. USA 83, 3068-3071 (1986)

3. Dong, C.: Vertex algebras associated with even lattices. J. Algebra 160, 245-265 (1993)

4. Dong, C., Lam, C.H., Yamada, H.: $W$-algebras in lattice vertex operator algebras. In: Lie Theory and Its Applications in Physics VII, ed. by H.-D. Doebner, V. K. Dobrev, Proc. of the VII International Workshop, Varna, Bulgaria, 2007, Bulgarian J. Phys. 35 supplement, 25-35 (2008)

5. Dong, C., Lam, C.H., Yamada, H.: W-algebras related to parafermion algebras. J. Algebra 322, 2366$2403(2009)$

6. Dong, C., Lam, C.H., Wang, Q., Yamada, H.: The structure of parafermion vertex operator algebras. J. Algebra 323, 371-381 (2010)

7. Dong, C., Lepowsky, J.: Generalized Vertex Algebras and Relative Vertex Operators. Progress in Math., Vol. 112, Boston, MA: Birkhäuser, 1993

8. Dong, C., Mason, G.: On quantum Galois theory. Duke Math. J. 86, 305-321 (1997)

9. Frenkel, I.B., Kac, V.: Basic representations of affine Lie algebras and dual resonance models. Invent. Math 62, 23-66 (1980)

10. Frenkel, I.B., Lepowsky, J., Meurman, A.: Vertex Operator Algebras and the Monster. Pure and Applied Math., Vol. 134, Boston, MA: Academic Press, 1988

11. Frenkel, I.B., Zhu, Y.: Vertex operator algebras associated to representations of affine and Virasoro algebras. Duke Math. J. 66, 123-168 (1992)

12. Gepner, D.: New conformal field theory associated with Lie algebras and their partition functions. Nucl. Phys. B290, 10-24 (1987)

13. Gepner, D., Qiu, Z.: Modular invariant partition functions for parafermionic field theories. Nucl. Phys. B285, 423-453 (1987)

14. Goddard, P., Kent, A., Olive, D.: Unitary representations of the Virasoro and super-Virasoro algebras. Commun. Math. Phys. 103, 105-119 (1986)

15. Hornfeck, K.: $W$-algebras with set of primary fields of dimensions $(3,4,5)$ and $(3,4,5,6)$. Nucl. Phys. B407, 237-246 (1993)

16. Humphreys, J.: Introduction to Lie Algebras and Representation Theory. New York: Spring-Verlag, 1987

17. Kac, V.G.: Infinite-dimensional Lie Algebras. $3^{\text {rd }}$ ed., Cambridge: Cambridge University Press, 1990

18. Lepowsky, J., Li, H.: Introduction to Vertex Operator Algebras and Their Representations. Progress in Math., Vol. 227, Boston, MA: Birkhäuser, 2004

19. Lepowsky, J., Primc, M.: Structure of the standard modules for the affine Lie algebra $A_{1}^{(1)}$. Contemp. Math. 46, Providence, RI: Amer. Math. Soc., 1985

20. Lepowsky, J., Wilson, R.L.: A new family of algebras underlying the Rogers-Ramanujan identities and generalizations. Proc. Natl. Acad. Sci. USA 78, 7245-7248 (1981)

21. Lepowsky, J., Wilson, R.L.: The structure of standard modules, I: Universal algebras and the RogersRamanujan identities. Invent. Math. 77, 199-290 (1984)

22. $\mathrm{Li}, \mathrm{H} .:$ An approach to tensor product theory for representations of a vertex operator algebra, $\mathrm{Ph} . \mathrm{D}$. thesis, Rutgers University, 1994

23. Meurman, A., Primc, M.: Vertex operator algebras and representations of affine Lie algebras. Representation of Lie groups, Lie algebras and their quantum analogues. Acta Appl. Math. 44, 207-215 (1996)

24. Wang, P., Ding, X.: $W$-algebra constructed from $\mathbb{Z}_{k}$-parafermion through Nahm's normal ordering product. Comm. Theor. Phys. 4, 155-189 (1995)

25. Wang, W.: Rationality of Virasoro vertex operator algebras. Int. Math. Res. Notices 7, 197-211 (1993)

26. West, P.: $W$ strings and cohomology in parafermionic theories. Phys. Lett. B 329, 199-209 (1994)

27. Zamolodchikov, A.B., Fateev, V.A.: Nonlocal (parafermion) currents in two-dimensional conformal quantum field theory and self-dual critical points in $Z_{N}$-symmetric statistical systems. Sov. Phys. JETP 62, 215$225(1985)$

Communicated by Y. Kawahigashi 